\title{
Entropic destruction of heavy quarkonium with hyperscaling violation
}

\author{
Ang $\mathrm{Li}^{1}$ and Zi-qiang Zhang, ${ }^{1,2}$ * \\ ${ }^{1}$ School of Mathematics and Physics, China University of Geosciences, Wuhan 4300\%, China \\ ${ }^{2}$ Key Laboratory of Quark and Lepton Physics (MOE), \\ Central China Normal University, Wuhan 430079, China
}

\begin{abstract}
We study the entropic destruction of heavy quarkonium in strongly coupled theories with an anisotropic scaling symmetry in time and spatial direction. We consider Lifshitz and hyperscaling violation theories which are covariant under a generalized Lifshitz scaling symmetry with the dynamical exponent $z$ and hyperscaling violation exponent $\theta$. It is shown that the entropic force depends on the parameters of these theories. In particular, increasing $z$ decreases the entropic force thus reducing the quarkonium dissociation, while increasing $\theta$ has opposite effect.
\end{abstract}

PACS numbers: 11.25.Tq, 11.15.Tk, 11.25-w

\section{INTRODUCTION}

It is believed that the heavy ion collisions at Relativistic Heavy Ion Collider (RHIC) and Large Hadron Collider (LHC) have produced a new state of matter so-called strongly coupled quark gluon plasma (QGP) [1 $[3]$. One of the signatures of QGP formation is the dissociation of heavy quarkonium [4]. Previous research has indicated that the immediate cause of this dissociation is Debye screening. However, there is a puzzle revealed in the recent experiment of charmonium $(c \bar{c})$ : the $c \bar{c}$ suppression at RHIC (lower energy density) was stronger than that at LHC (larger energy density) [5, [6]. Obviously, this finding is in conflict with the Debye screening and the thermal activation [7, 8]. To explain this, D. E Kharzeev argued [9] that an anomalously strong suppression of $c \bar{c}$ near the deconfinement transition could be a consequence of the nature of deconfinement. This inference was based on the lattice QCD results 10-13] which indicated a large amount of entropy $S$ associated with the heavy quarkonium (or $q \bar{q}$ ) in QGP and this entropy was found to grow as a function of the distance between $q \bar{q}$. In the proposal of [9] the entropy gives rise to the entropic force

$$
\mathcal{F}=T \frac{\partial S}{\partial L},
$$

where $T$ is the temperature of the plasma and $L$ is the inter-quark distance of $q \bar{q}$. It has been conjectured [9] that the entropic force is the leading role in the deconfinement transition itself and a possible relation of the observed peak in the entropy near the deconfinement transition to the long string condensation 14 22].

AdS/CFT correspondence [23 25], which maps a $d$ dimensional quantum field theory to its dual gravitational theory, living in $d+1$ dimensional, has yielded many important insights for studying different aspects of QGP [26]. Using AdS/CFT, D. Kharzeev first calculated the entropic force for $\mathcal{N}=4$ super Yang-Mills (SYM) plasma [27]. Therein, it was found that the narrow and strong peak in the entropy near the transition temperature is related to the deconfinement of quarkonium. Soon after, this idea was extended to various cases, e.g., the entropic force of moving quarkonium was investigated in [28]. The chemical potential effect on the entropic force was studied in [29]. Also, this quantity has been addressed from AdS/QCD [30]. Other related results can be found in [31 33].

In this paper, we are interested in studying the entropic force in strongly coupled theories with an anisotropic scaling symmetry in time and spatial direction. In particular, we will consider hyperscaling violation theories [34 38] which are covariant under a generalized Lifshitz scaling symmetry with the dynamical exponent $z$ and hyperscaling violation exponent $\theta$ and apply their gravity duals. Because of different scaling of time and space, these theories are intrinsically non-relativistic. On the other hand, the metrics of these theories are scale invariant but not conformally invariant. From a QCD point of view, such theories could be used as a basis for new AdS/QCD constructions. Recently, the hyperscaling violation theories have been used to describe the string theory [39 43] and holographic superconductors [44 47]. Also, some QCD observables or quantities, e.g., Schwinger effect [48], heavy quark potential [49, 50] have been addressed in such theories. Motivated by this, in this paper, we study the entropy force in hyperscaling violation theories. Specifically, we want to understand how the non-relativistic parameters $z$ and $\theta$ modify the entropy force as well as the quarkonium dissociation.

*Electronic address: zhangzq@cug.edu.cn 
The paper is organized as follows. In the next section, we introduce the hyperscaling violation theories given in [43]. In section 3, we study the behavior of the entropic force with respect to a heavy quarkonium in these theories and analyze the effects of $z$ and $\theta$ on it. In section 4 , we summarize the results and make some discussions.

\section{HYPERSCALING VIOLATION BACKGROUND}

Let's start with a brief review of the backgrounds with hyperscaling violation [43]. The corresponding metric takes the form [51, 52]

$$
d s^{2}=u^{\theta}\left[-\frac{d t^{2}}{u^{2 z}}+\frac{b_{0} d u^{2}+d x^{i} d x^{j}}{u^{2}}\right]
$$

and is invariant under a generalized Lifshitz scaling $t \rightarrow \Lambda^{z} t, u \rightarrow \Lambda u, x^{i} \rightarrow \Lambda x^{i}, d s^{2} \rightarrow \Lambda^{-\theta} d s^{2}$, where $b_{0}=\ell^{2}$ with $\ell$ the IR scale. $z$ is the dynamical Lifshitz parameter (or is called the dynamical critical exponent) which characterizes the behavior of system near the phase transition. $\theta$ denotes the hyperscaling violation exponent which is responsible for the nonstand scaling of physical quantities and controls the transformation of the metric.

The scalar curvature is

$$
\mathcal{R}=-\frac{3 \theta^{2}-4(z+3) \theta+2\left(z^{2}+3 z+6\right)}{b_{0}} u^{-\theta} .
$$

Note that the geometry is flat for $\theta=2, z=0,1$. It is Ricci flat for $\theta=4, z=3$, and in Ridler coordinates for $\theta=0, z=1$.

Using a radical redefinition

$$
u=(2-z) r^{1 /(2-z)}
$$

and rescaling $x^{i}$ and $t$, one gets:

$$
d s^{2} \sim r^{\frac{\theta-2}{2-z}}\left[-f(r) d t^{2}+\frac{d r^{2}}{f(r)}+d x^{i} d x^{i}\right], \quad f(r)=f_{0}\left(\frac{r}{\ell}\right)^{\frac{2(1-z)}{2-z}},
$$

with $f_{0}=(2-z)^{2(1-z)}$. The boundary is at $r=\infty$ when $\frac{\theta-2}{2-z}<0$ and at $r=0$ when $\frac{\theta-2}{2-z}>0$.

The energy scale reads

$$
E \simeq u^{(\theta-2 z) / 2} \simeq r^{(\theta-2 z) / 2(2-z)}
$$

As discussed in [43], the metrics (5) satisfy the Gubser conditions (in the context of holography, many non-trivial flows become singular upon dimensional reduction, leading to the Gubser criterion for the acceptability of a naked singularity [53]), given by

$$
\frac{2 z+3(2-\theta)}{2(z-1)-\theta}>0, \quad \frac{z-1}{2(z-1)-\theta}>0, \quad \frac{2(z-1)+3(2-\theta)}{2(z-1)-\theta}>0,
$$

and the thermodynamic stability condition

$$
\frac{z}{2(z-1)-\theta}>0
$$

The generalizations of (5) to include finite temperature are

$$
d s^{2} \sim\left(\frac{r}{\ell}\right)^{-\alpha}\left[-f(r) d t^{2}+\frac{d r^{2}}{f(r)}+d x^{i} d x^{i}\right]
$$

with

$$
f(r)=f_{0}\left(\frac{r}{\ell}\right)^{2 \beta}\left[1-\left(\frac{r}{r_{h}}\right)^{\gamma}\right]
$$

where

$$
\alpha=\frac{\theta-2}{z-2}, \quad \beta=\frac{z-1}{z-2}, \quad \gamma=\frac{2 z+3(2-\theta)}{2(2-z)} .
$$

The Hawking temperature reads

$$
T=\frac{f_{0}}{8 \pi \ell}\left(\frac{r_{h}}{\ell}\right)^{\frac{z}{z-2}}\left|\frac{2 z-3 \theta+6}{z-2}\right| .
$$

For more about the hyperscaling violation backgrounds, we refer to [43]. 


\section{ENTROPIC FORCE}

We now proceed to holographically compute the entropic force for the background metric (9) following [27]. The Nambu-Goto action is

$$
S_{N G}=T_{F} \int d \tau d \sigma \mathcal{L}=-\frac{1}{2 \pi \alpha^{\prime}} \int d \tau d \sigma \sqrt{-\operatorname{det} g_{\alpha \beta}},
$$

where $T_{F}=\frac{1}{2 \pi \alpha^{\prime}}$ is the fundamental string tension and $\alpha^{\prime}$ is related to the 't Hooft coupling $\lambda$ by $\frac{R^{2}}{\alpha^{\prime}}=\sqrt{\lambda}$ (hereafter we set $R=1) . g$ represents the determinant of the induced metric with

$$
g_{\alpha \beta}=g_{\mu \nu} \frac{\partial X^{\mu}}{\partial \sigma^{\alpha}} \frac{\partial X^{\nu}}{\partial \sigma^{\beta}}
$$

where $X^{\mu}$ and $g_{\mu \nu}$ are the target space coordinates and metric, respectively.

For our purpose, we take the static gauge

$$
t=\tau, \quad x=\sigma, \quad y=0, \quad z=0,
$$

and assume $r$ depends only on $\sigma$

$$
r=r(\sigma)
$$

given that, the induced metric becomes

$$
\left.g_{00}=-b(r) f(r), \quad g_{01}=g_{10}=0, \quad g_{11}=b(r)\left[1+\frac{\dot{r}}{f(r)}\right)\right],
$$

with $b(r)=(r / \ell)^{-\alpha}, \dot{r}=d r / d \sigma$, then the Lagrangian density reads

$$
\mathcal{L}=\sqrt{b^{2}(r)\left[f(r)+\dot{r}^{2}\right]} .
$$

Since $\mathcal{L}$ does not depend on $\sigma$ explicitly, the corresponding Hamiltonian is a constant, that is

$$
\mathcal{H}=\frac{\partial \mathcal{L}}{\partial \dot{r}} \dot{r}-\mathcal{L}=\text { Constant }
$$

Imposing the boundary condition at the tip of the U-shaped string

$$
\dot{r}=0, \quad r=r_{c} \quad\left(r_{h}>r_{c}\right),
$$

one gets

$$
\dot{r}=\sqrt{\frac{f^{2}(r) b^{2}(r)-f(r) f\left(r_{c}\right) b^{2}\left(r_{c}\right)}{f\left(r_{c}\right) b^{2}\left(r_{c}\right)}},
$$

with

$$
f\left(r_{c}\right)=f_{0}\left(\frac{r_{c}}{\ell}\right)^{2 \beta}\left[1-\left(\frac{r_{c}}{r_{h}}\right)^{\gamma}\right], \quad b\left(r_{c}\right)=\left(\frac{r_{c}}{\ell}\right)^{-\alpha} .
$$

The inter-distance of $q \bar{q}$ can be calculated by integrating (21)

$$
L=2 \int_{0}^{r_{c}} d r \sqrt{\frac{A\left(r_{c}\right) B(r)}{A^{2}(r)-A(r) A\left(r_{c}\right)}} .
$$

with

$$
A(r)=b^{2}(r) f(r), \quad B(r)=b^{2}(r), \quad A\left(r_{c}\right)=b^{2}\left(r_{c}\right) f\left(r_{c}\right),
$$

the shape of $L$ against $\varepsilon$ with $\varepsilon=r_{c} / r_{h}$ is shown in Fig. 1. Here we take $z=1.6, \theta=1$ (other values of $z$ and $\theta$ lead to similar picture). 


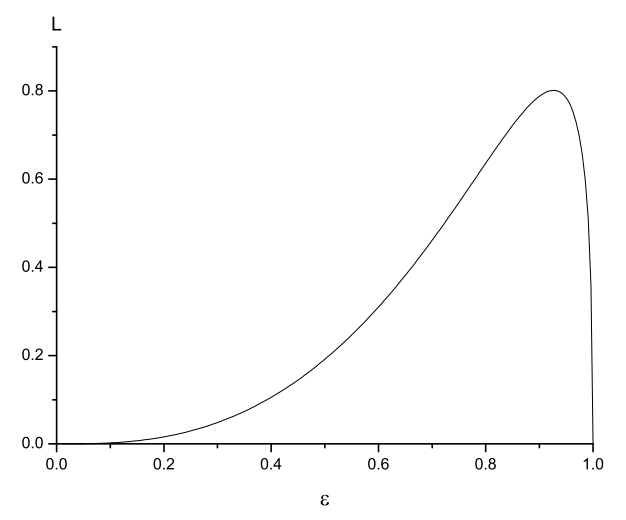

FIG. 1: $L$ versus $\varepsilon$. Here $z=1.6, \theta=1$.

The next task is to calculate entropy, given by

$$
S=-\frac{\partial F}{\partial T},
$$

where $F$ is the free energy of the $q \bar{q}$, which is related to the on-shell action of the fundamental string in the dual geometry. Note that this quantity has been holographically studied at zero temperature [54] and finite temperature [55, 56]. Broadly speaking, there are two cases for $F$.

1. If $L>\frac{c}{T}$ ( $c$ denotes the maximum value of $\left.L T\right)$, some new configurations should be considered [57] and then there are a few possible choices of $F$ [58], e.g., one may choose a configuration of two disconnected trailing drag strings [59, 60]. Under such conditions, the free energy is

$$
F^{(1)}=\frac{1}{\pi \alpha^{\prime}} \int_{0}^{r_{c}} d r
$$

leading to

$$
S^{(1)}=\sqrt{\lambda} \theta\left(L-\frac{c}{T}\right)
$$

where $\theta\left(L-\frac{c}{T}\right)$ is the Heaviside step function.

2. If $L<\frac{c}{T}$, the fundamental string is connected, then the free energy of the $q \bar{q}$ could be obtained by plugging (21) into (18), that is

$$
F^{(2)}=\frac{1}{\pi \alpha^{\prime}} \int_{0}^{r_{c}} \sqrt{\frac{A(r) B(r)}{A(r)-A\left(r_{c}\right)}},
$$

then from (12), (25) and (28), one gets

$$
S^{(2)}=-\frac{\partial F^{(2)}}{\partial T}=-\frac{\partial F^{(2)}}{\partial r_{h}} / \frac{\partial T}{\partial r_{h}}
$$

where

$$
\begin{gathered}
\frac{\partial F^{(2)}}{\partial r_{h}}=\frac{1}{2 \pi \alpha^{\prime}} \int_{0}^{r_{c}} \frac{A^{\prime}(r) B(r)\left[A(r)-A\left(r_{c}\right)\right]-A(r) B(r)\left[A^{\prime}(r)-A^{\prime}\left(r_{c}\right)\right]}{\sqrt{A(r) B(r)\left[A(r)-A\left(r_{c}\right)\right]^{3}}}, \\
\frac{\partial T}{\partial r_{h}}=\frac{f_{0}}{8 \pi \ell}\left|\frac{2 z-3 \theta+6}{z-2}\right| \ell^{\frac{z-2}{z}} \frac{z}{z-2} r_{h}^{\frac{2}{z-2}}
\end{gathered}
$$

with

$$
\begin{aligned}
A^{\prime}(r) & =b^{2}(r) f_{0}\left(\frac{r}{\ell}\right)^{2 \beta} \gamma r^{\gamma} r_{h}^{-\gamma-1}, \\
A^{\prime}\left(r_{c}\right) & =\left.A^{\prime}(r)\right|_{r=r_{c}},
\end{aligned}
$$


note that by plugging $z=1$ and $\theta=0$ in (29), the entropic force for $\mathcal{N}=4 \mathrm{SYM}$ [27] can be reproduced, as expected.

Next, we calculate the entropy force. Before we get to that, we determine the value range of $z$ and $\theta$. According to the Gubser condition of (77) and the thermodynamic stability of (8), one has

$$
1<z<2, \quad \theta<2, \quad \theta<z .
$$

Taken together, one can numerically calculate the entropic force from (29). In Fig.2, we plot $S^{(2)} / \sqrt{\lambda}$ versus $L T$ for different values of $z$ and $\theta$, here we have used the relation $\frac{1}{\alpha^{\prime}}=\sqrt{\lambda}$. From the left panel, one can see that with fixed $z$, increasing $\theta$ leads to larger entropy at small distances. While from the right panel one finds that with fixed $\theta$, increasing $z$ leads to smaller entropy at small distances. As stated earlier, the entropic force is found to grow as a function of the distance (see (1D) and responsible for the quarkonium dissociation. Thus, one concludes that increases $\theta$ increases the entropic force thus enhancing the quarkonium dissociation, while $z$ has opposite effect.

To analyze how the temperature influences the entropic force in the hyperscaling violation backgrounds, we plot $S^{(2)} / \sqrt{\lambda}$ versus $L$ with fixed $z$ and $\theta$ in Fig.3. One finds increasing $T$ leads to increasing the entropic force, in accord with the finding in [61]. The physical meaning of our results will be discussed in the next section.
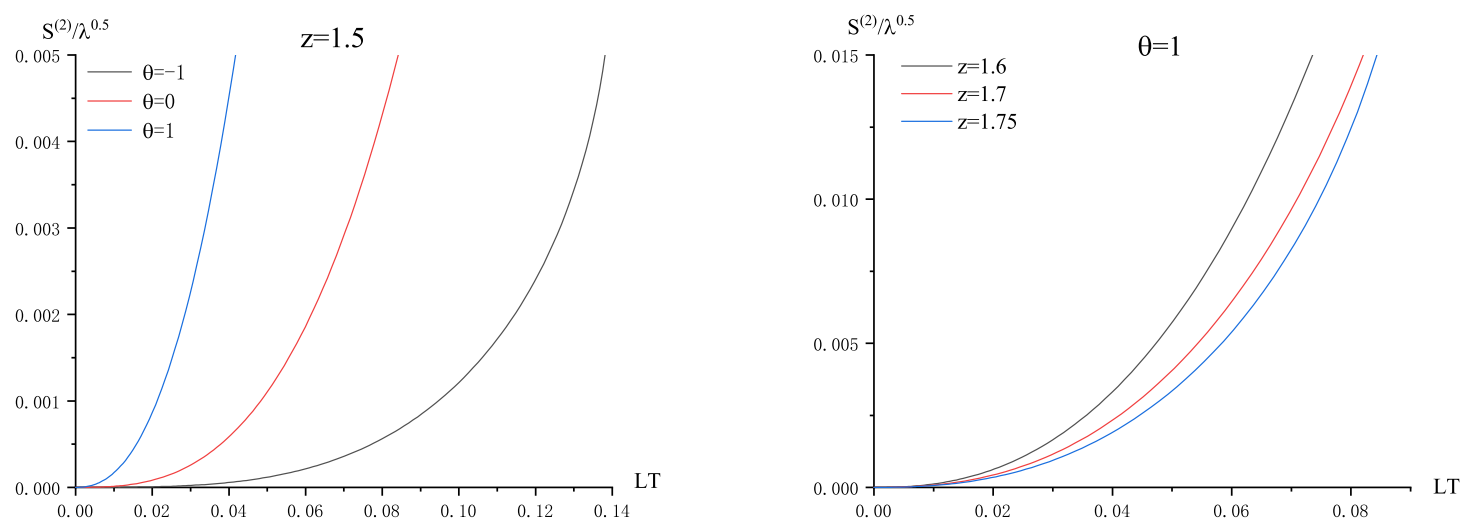

FIG. 2: $S^{(2)} / \sqrt{\lambda}$ versus $L T$ for different $\theta$ and $z$. Left: $z=1.5$ and from left to right $\theta=1,0,-1$. Right: $\theta=1$ and from left to right $z=1.6,1.7,1.75$.

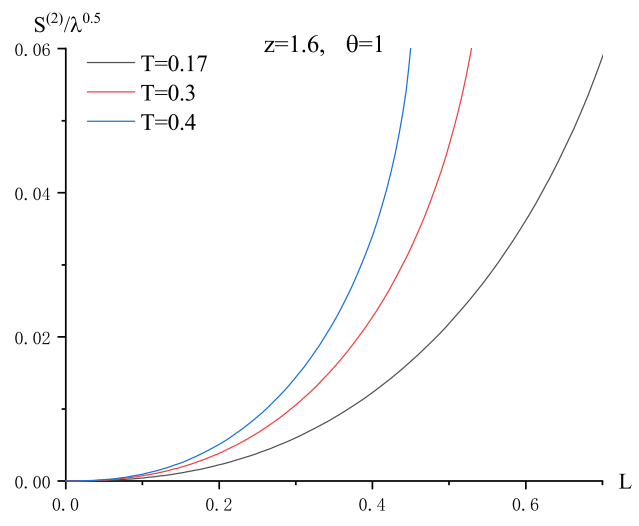

FIG. 3: $S^{(2)} / \sqrt{\lambda}$ versus $L$. Here $z=1.6, \theta=1$, and from top to bottom $T=0.4,0.3,0.17$, respectively. 


\section{CONCLUSION AND DISCUSSION}

In this paper, we studied the entropic destruction of heavy quarkonium in the non-relativistic backgrounds. We considered Lifshitz and hyperscaling violation theories which are strongly coupled theories with an anisotropic scaling symmetry in the time and a spatial direction. An understanding of how the entropic force changes by these theories may be essential for theoretical predictions. We discussed how the entropic force depends on the non-relativistic parameters $z$ and $\theta$. It is shown that increasing $z$ decreases the entropic force thus reducing the quarkonium dissociation. Namely, the quarkonium melts harder in the presence of an anisotropic scaling symmetry in the time and a spatial direction. On the other hand, we find that increases $\theta$ increases the entropic force, indicating the inclusion of $\theta$ (means hyperscaling violation in the dual field theory) enhances the quarkonium dissociation.

Here we would like to point out that the heavy quarkonium discussed here mainly refers to charmonium. Because most of the bottomonium $(b \bar{b})$ has smaller sizes, and is thus much less affected by the entropic force 31]. Actually, the available data shows that the $b \bar{b}$ suppression is indeed stronger at LHC [62, 63] than at RHIC [64, 65].

Finally, it is relevant to mention that the imaginary potential [66] has been proposed to responsible for melting the heavy quarkonium recently. It would be interesting to investigate this quantity in hyperscaling violation theories as well. We leave this for further study.

\section{ACKNOWLEDGMENTS}

This work is supported by the Innovation Fund of of Key Laboratory of Quark and Leption Physics under grant No. QLPL2020P01.

[1] J. Adams et al. [STAR Collaboration], Nucl. Phys. A 757, 102 (2005).

[2] K. Adcox et al. [PHENIX Collaboration], Nucl. Phys. A 757, 184 (2005).

[3] E. V. Shuryak, Nucl. Phys. A 750, 64 (2005).

[4] T. Matsui, H. Satz, Phys. Lett. B 178, 416 (1986).

[5] A. Adare et al. (PHENIX Collaboration), Phys. Rev. Lett. 98, 232301 (2007).

[6] B. B. Abelev et al. (ALICE Collaboration), Phys. Lett. B 734, 314 (2014).

[7] D. Kharzeev and H. Satz, Phys. Lett. B 334, 155 (1994).

[8] E. V. Shuryak, Phys. Lett. B 78, 150 (1978).

[9] D. E. Kharzeev, Phys. Rev. D 90, 074007 (2014).

[10] O. Kaczmarek, F. Karsch, P. Petreczky, and F. Zantow, Phys. Lett. B 543, 41 (2002).

[11] O. Kaczmarek and F. Zantow, arxiv: 0506019 [hep-lat].

[12] P. Petreczky and K. Petrov, Phys. Rev. D 70, 054503 (2004).

[13] O. Kaczmarek and F. Zantow, PoS LAT 2005, 192 (2006).

[14] J. Kogut, L. Susskind, Phys. Rev. D, 1975, 11(2): 395.

[15] R. D. Pisarski, O. Alvarez, Phys. Rev. D, 1982, 26(12): 3735.

[16] A. Patel , Nucl. Phys. B 243 (1984) 411-422

[17] Y. Aharonov, F. Englert, J. Orloff, Phys. Lett. B 199 (1987) 366-370.

[18] N. Deo, S. Jain, C. I. Tan, Phys. Rev. D, 1989, 40(8): 2626.

[19] M. A. Levin, X. G. Wen, Phys. Rev. B, 2005, 71(4): 045110.

[20] T. Kalaydzhyan, E. Shuryak, Phys. Rev. D, 2014, 90(2): 025031.

[21] M. Hanada, J. Maltz, L. Susskind - Phys. Rev. D, 2014, 90(10): 105019.

[22] K. Hashimoto, S. Kinoshita, K. Murata, T. Oka, Phys. Lett. B 746 (2015) 311-314.

[23] J. Maldacena, Int. J. Theor. Phys. 38 (1999) 1113-1133.

[24] S. S. Gubser, I. R. Klebanov, A. M. Polyakov, Phys. Lett. B 428 (1998) 105-114.

[25] E. Witten, Adv.Theor.Math.Phys. 2 (1998) 253-291.

[26] J. C. Solana, H. Liu, D. Mateos, K. Rajagopal, and U. A. Wiedemann, arXiv:1101.0618 [hep-th].

[27] K. Hashimoto and D. E. Kharzeev, Phys. Rev. D 90, 125012 (2014).

[28] K. B. Fadafan, S. K. Tabatabaei, Phys. Rev. D 94, 026007 (2016).

[29] Z. q. Zhang, D. f. Hou, and G. Chen, Phys. Lett. B 768, 180 (2017).

[30] I. Iatrakis and D. E. Kharzeev, Phys. Rev. D 93, 086009 (2016).

[31] D. E. Kharzeev, Nucl. Part. Phys. Proc. 276-278 (2016) 90-95.

[32] Z. q. Zhang, Phys. Rev. D 101 (2020) 10, 106005.

[33] Z. q. Zhang, D. f. Hou, Phys. Lett. B 803 (2020) 135301

[34] T. Azeyanagi, W. Li, T. Takayanagi, JHEP 06 (2009) 084.

[35] K. Balasubramanian, K. Narayan, JHEP 08 (2010) 014. 
[36] H. Singh, JHEP 12 (2020) 082.

[37] K. Narayan, Phys. Rev. D, 2012, 85(10): 106006.

[38] P. Dey, S. Roy, Phys. Rev. D, 2012, 86(6): 066009.

[39] B. Goutraux, E. Kiritsis, JHEP 12 (2011) 036.

[40] E. Perlmutter, JHEP 02 (2011) 013.

[41] M. Alishahiha, H. Yavartanoo, JHEP 11 (2012) 034.

[42] J. Sadeghi, B. Pourhassan, F. Pourasadollah, Phys. Lett. B 720 (2013) 244-249.

[43] E. Kiritsis, JHEP 01 (2013) 030.

[44] E. J. Brynjlfsson, U. H. Danielsson, et al. J. Phys. A 43 (2010) 065401.

[45] S. J. Sin, S. S. Xu, Y. Zhou, Int. J. Mod. Phys. A 26 (2011) 4617-4631.

[46] Y. Bu, Phys. Rev. D 86 (2012) 046007.

[47] Q. Pan, S. J. Zhang, Eur. Phys. J. C 76 (2016) 3, 126.

[48] K. B. Fadafana, F. Saiedi, Eur. Phys. J. C (2015) 75:612.

[49] S. Tahery, J. Sadeghi, J. Phys. G 44 (2017) 10, 105001.

[50] Z. q. Zhang, C. Ma, D. f. Hou, G. Chen, Adv. High Energy Phys. 2017 (2017) 8276534.

[51] C. Charmousis, B. Gouteraux, B.S. Kim, E. Kiritsis and R. Meyer, JHEP 11 (2010) 151.

[52] B. Gouteraux and E. Kiritsis, JHEP 12 (2011) 036.

[53] S. S. Gubser, Adv. Theor. Math. Phys. 4 (2000) 679.

[54] J. M. Maldacena, Phys. Rev. Lett. 80 (1998) 4859.

[55] A. Brandhuber, N. Itzhaki, J. Sonnenschein, S. Yankielowicz, Phys. Lett. B 434 (1998) 36.

[56] S. J. Rey, S. Theisen, J.T. Yee, Nucl. Phys. B 527 (1998) 171.

[57] D. Bak, A. Karch, L.G. Yaffe, JHEP 0708 (2007) 049.

[58] M. Chernicoff, J.A. Garcia, A. Guijosa, JHEP 0609 (2006) 068.

[59] C. P. Herzog, A. Karch, P. Kovtun, JHEP 07 (2006) 013.

[60] S. S. Gubser, Phys. Rev. D, 2006, 74(12): 126005.

[61] Z. q. Zhang, D. f. Hou, Phys. Lett. B 803 (2020) 135301.

[62] S. Chatrchyan et al. [CMS Collaboration], Phys. Rev. Lett. 109, 222301 (2012).

[63] B. B. Abelev et al. [ALICE Collaboration], Phys. Lett. B 738, (2014) 361-372.

[64] L. Adamczyk et al. [STAR Collaboration], Phys. Lett. B 735, 127 (2014).

[65] A. Adare et al. [PHENIX Collaboration], Phys.Rev.C 91 (2015) 2, 024913.

[66] J. Noronha, A. Dumitru, Phys. Rev. Lett. 103 (2009) 152304 